\title{
A small, well-differentiated rectal neuroendocrine tumor with multiple lymph node metastases: A case report
}

\author{
SEUNG-JOO NAM ${ }^{1}$, GI BONG CHAE ${ }^{2}$, SEUNGKOO LEE ${ }^{3}$, SUNG CHUL PARK $^{1}$, \\ CHANG DON KANG ${ }^{1}$ and SUNG JOON LEE ${ }^{1}$ \\ Departments of ${ }^{1}$ Internal Medicine, ${ }^{2}$ Surgery and ${ }^{3}$ Pathology, Kangwon National University School of Medicine, \\ Chuncheon, Gangwon 24289, Republic of Korea
}

Received August 4, 2017; Accepted January 29, 2018

DOI: $10.3892 / \mathrm{ol} .2018 .8257$

\begin{abstract}
The incidence of rectal neuroendocrine tumor (NET), which is often diagnosed during routine surveillance endoscopy, is increasing. The majority of these tumors are small and asymptomatic, possessing benign features with favorable prognoses. At present, small rectal NETs without high-risk factors are typically treated by local resection, including endoscopic mucosal resection, endoscopic submucosal dissection, or transanal endoscopic microsurgery, with or without additional imaging follow-up by abdominal computed tomography or magnetic resonance imaging. The present study, however, describes a case of a small rectal NET without any known risk factors, which was accompanied by substantial locoregional lymph node metastasis, underscoring the importance of imaging studies for rectal NETs.
\end{abstract}

\section{Introduction}

The incidence and prevalence of gastroenteropancreatic neuroendocrine tumors (NETs) have been increasing globally; the crude incidence is estimated to be $5.25 / 100000 /$ year and the prevalence to be $35 / 100000 /$ year according to the 2012 European Society of Medical Oncology guidelines (1), and rectal NETs are the most common type of gastroenteropancreatic NET in Asia, according to the epidemiological reports in 2010 and 2012 (2,3). Among rectal NETs, 50\% of cases are asymptomatic and are identified incidentally during routine surveillance colonoscopy as sub-epithelial tumors (4). Little is known with regard to the risk factors of rectal NETs due to the lack of epidemiological studies. However, a small number of studies have proposed potential risk factors for rectal NETs, including sex, age, ethnicity, general family history of cancer

Correspondence to: Professor Gi Bong Chae, Department of Surgery, Kangwon National University School of Medicine, 156 Baengnyeong-ro, Chuncheon, Gangwon 24289, Republic of Korea E-mail: cgb3377@kangwon.ac.kr

Key words: neuroendocrine tumor, rectum, lymph node, metastasis and alcohol consumption (5-7). Men exhibited a higher risk of rectal NETs compared with women in a study of an Asian population, whereas female predominance was revealed in populations from the USA or Sweden, indicating possible differences in susceptibility to rectal NETs according to the ethnic group (5-7). In addition, non-Caucasian populations (those of Hispanic, African or Asian descent) exhibited a higher prevalence of rectal NETs compared with Caucasian populations (6). Young age $(<50$ or $\leq 60)$ was also associated with an increased risk of developing rectal NET $(5,6)$. Furthermore, there are conflicting data regarding alcohol and smoking exposure, but a previous study suggested alcohol consumption as an important risk factor (5). However, due to the lack of large epidemiological studies, the strength of these associations is not fully known, and additional studies to determine the risks associated with rectal NETs are required.

The majority of rectal NETs are small and superficial, with indolent characteristics (8). Traditionally, tumor size and depth of invasion are considered important risk factors for metastasis. Due to their low risk of metastasis, the standard treatment modality for small $(<2 \mathrm{~cm})$ and superficial (confined within submucosa) rectal NETs is local resection, including endoscopic mucosal resection (EMR), cap-assisted EMR (C-EMR), endoscopic submucosal dissection (ESD), and transanal endoscopic microsurgery $(4,9)$. However, several studies have described the occurrence of lymph node metastasis from these small tumors, resulting in disagreement over the optimal treatment modality (local resection vs. radical resection) (10). Some authors have recommended a stratified approach outlined by the study of Fahy et al (11), which assigns a score after considering risk factors for poor outcome, e.g. distant metastasis, recurrent disease and reduced recurrence-free and diseases-specific survival. This also takes into consideration tumor size, mitotic rate, deep invasion and lymphovascular invasion, which can be measured by an endoscopic biopsy sample or endoscopic excision (e.g., EMR or ESD) (11). In case of low risk tumors (for poor outcome), endoscopic or transanal excision may be acceptable; however, in cases of high-risk tumors, radical resection should be considered (11).

The present study describes the case of a small rectal NET, without any known risk factors, but with substantial locoregional lymph node metastasis. 

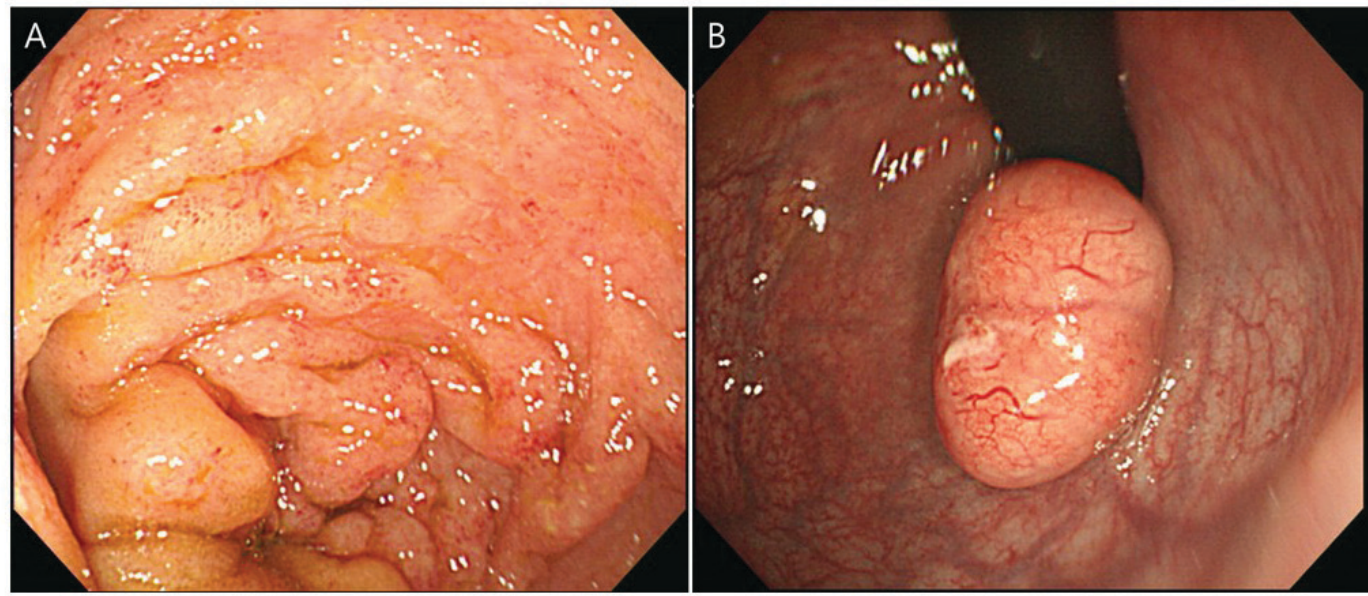

Figure 1. Colonoscopic image of terminal ileum and distal rectum. (A) Terminal ileitis with edematous hyperemic mucosa. (B) Oval-shaped subepithelial tumor with normal surface mucosa and smooth margins in the distal rectum.

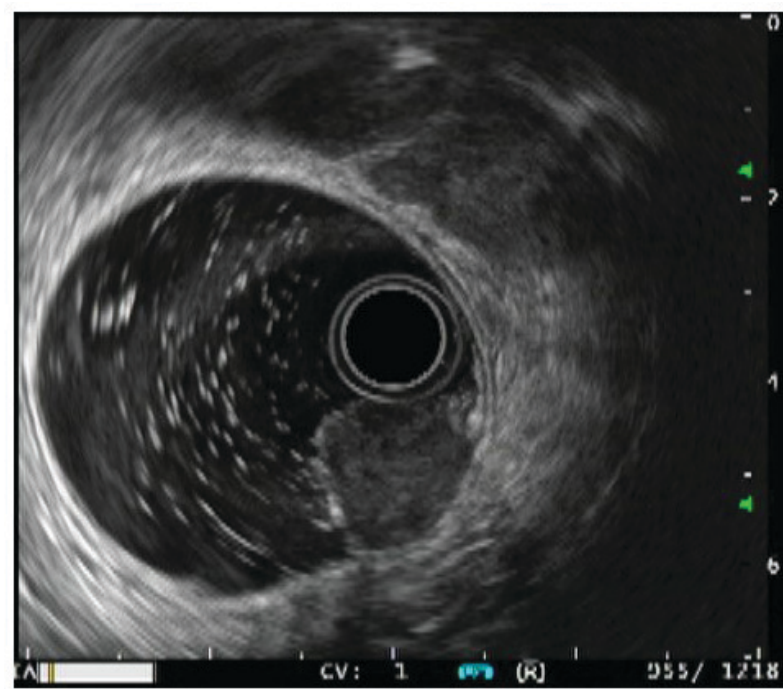

Figure 2. Transrectal endoscopic ultrasound revealing a $1.5-\mathrm{cm}$, ovoid, hypoechoic, subepithelial lesion with smooth margins originating from the second layer, which corresponds to deep mucosal layer.

\section{Case report}

A 25-year-old man presented to Kangwon National University Hospital (Chuncheon, South Korea) in March 2017, subsequent to suffering from acute diarrhea, fever, vomiting and abdominal pain for 3 days, in addition to 2 years of chronic anal bleeding. A physical examination revealed mild abdominal tenderness in his right lower quadrant. A digital rectal examination indicated a $1-\mathrm{cm}$, hard, smooth-surfaced and palpable mass in the distal rectum. A sigmoidoscopy was performed to evaluate the palpable distal rectal lesion; the terminal ileum was reached following the removal of fecal material, which revealed edematous hyperemic mucosa of the terminal ileum and an oval-shaped subepithelial tumor (SET) with normal surface mucosa in the distal rectum. The colonic mucosa was otherwise intact, without inflammation or bleeding (Fig. 1). A transrectal endoscopic ultrasound (EUS) for distal rectal SET revealed a $1.5-\mathrm{cm}$, ovoid, hypoechoic lesion originating from the second layer, which corresponded to the deep mucosa (Fig. 2).

The most probable diagnosis was a rectal carcinoid tumor combined with acute gastroenteritis, and it was decided to pursue a histopathological diagnosis by endoscopic or surgical resection following resolution of the acute enteritis. Prior to resection, an abdominopelvic computed tomography (CT) scan performed to evaluate the distal rectal mass and terminal ileal inflammation; this indicated wall thickening of the terminal ileum with several mesenteric lymph nodes in the right lower quadrant, a $1.5-\mathrm{cm}$ nodule in the distal rectum with an enlarged lymph node in the right posterior mesorectum, and several enlarged nodes along the inferior rectal vessel and left para-aortic area (Fig. 3).

The condition of the patient was discussed with a surgeon to decide the management of multiple enlarged perirectal lymph nodes. Firstly, a transanal excision of the tumor was performed to allow pathological diagnosis, which revealed a grade 1 NET (according to the World Health Organization 2010 classification) (1), $1.5 \times 1.2 \mathrm{~cm}$ in size and confined to submucosa, with the following characteristics: Synaptophysin-positive, neural cell adhesion molecule (CD56)-positive, $<1$ mitosis per 10 high-power fields (HPF), <1\% Ki-67-positive cells, and no lymphovascular invasion (Fig. 4). Immunohistochemically, the neuroendocrine markers synaptophysin and CD56 were strong and diffuse throughout the whole tumor, consistent with a well-differentiated NET (Fig. 4). Immunohistochemical staining was performed on $5-\mu \mathrm{m}$-thick formalin-fixed (room temperature for $5 \mathrm{~h}$ ), paraffin-embedded tumor tissue sections using the BenchMark XT automated tissue staining system (Ventana Medical Systems, Inc., Tucson, AZ, USA) according to the manufacturer's protocol. The primary antibodies used were synaptophysin (cat. no., 790-4407; Ventana Medical Systems, pre-diluted and ready to use) and CD56 (cat. no., M7304; clone 123C3; Dako; Agilent Technologies, Inc., Santa Clara, CA, USA, dilution 1:100). Each were incubated with the samples for $30 \mathrm{~min}$ at $37^{\circ} \mathrm{C}$. All tissue slides were then incubated with a horseradish peroxidase-conjugated secondary antibody (ultraView Universal DAB Detection kit, Ventana Medical Systems; cat. no., 760-500, pre-diluted and ready to 

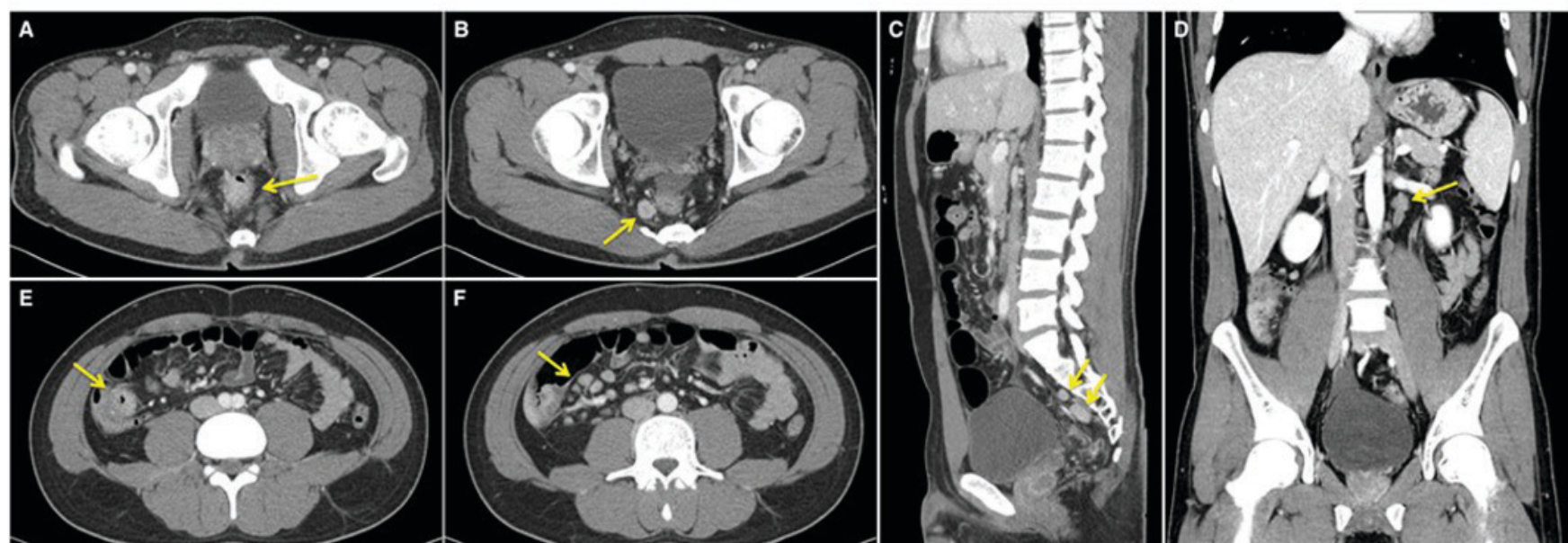

Figure 3. Abdominopelvic computed tomography scan indicating multiple enlarged lymph nodes. (A) 1.5-cm nodule in the distal rectum (arrow). (B) Enlarged lymph node in the right posterior mesorectum (arrow). (C) Arrows indicate several aggregated enlarged lymph nodes along the inferior rectal vessel. (D) Left para-aortic lymph node enlargement (arrow). Wall thickening of the (E) terminal ileum, with (F) several enlarged mesenteric lymph nodes in the right lower quadrant.
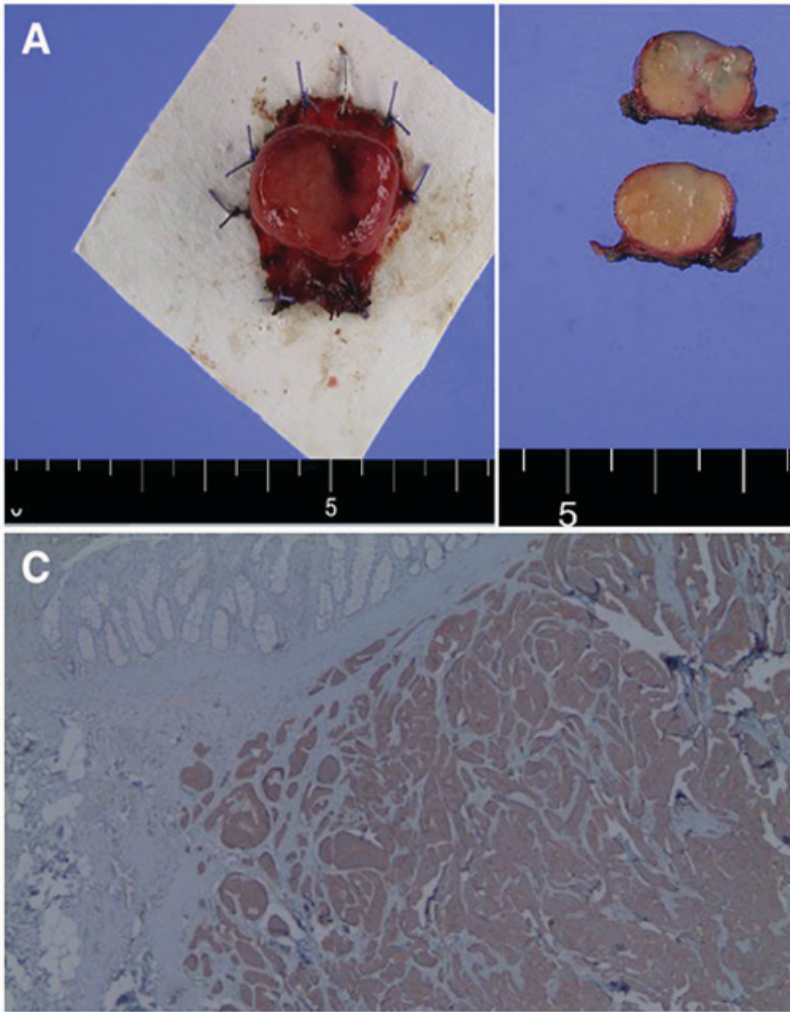
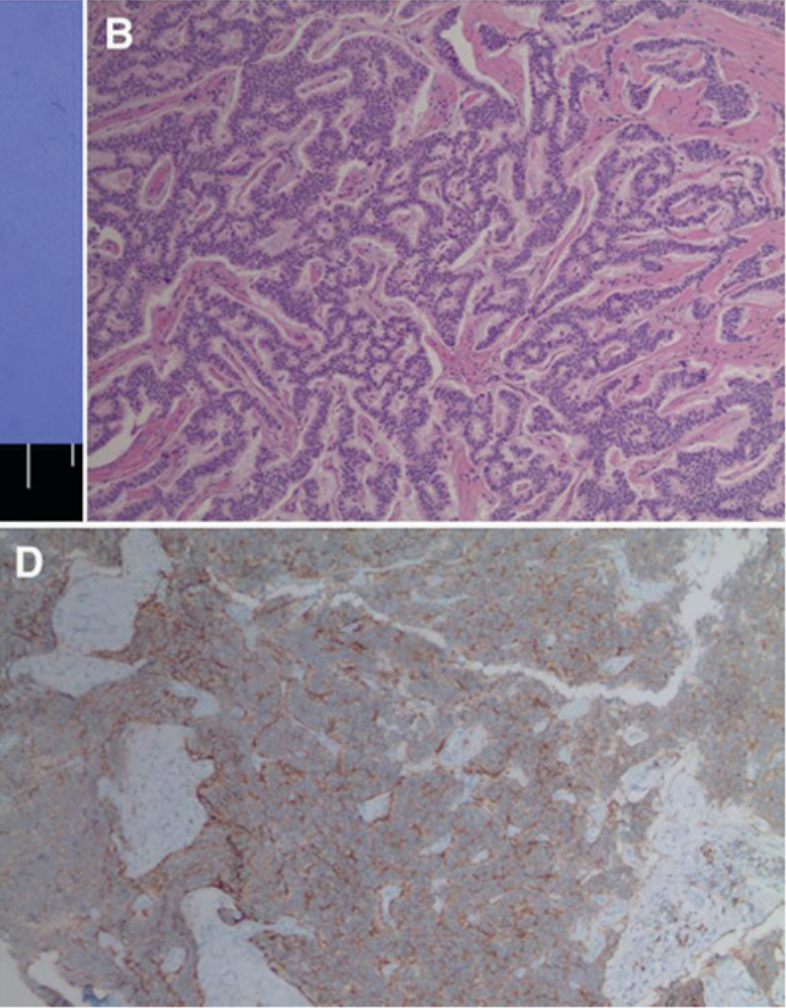

Figure 4. Distal rectal mass obtained through transanal excision. (A) Resected rectal mass, $1.5 \mathrm{~cm}$ in size. (B) Histological features showing a trabecular, ribbon-like growth pattern of round to oval-shaped tumor cells. No tumor necrosis was present. Hematoxylin and eosin staining; magnification, x200. (C) Immunohistochemical staining indicating the diffuse expression of synaptophysin throughout the whole tumor (magnification, x100). (D) Immunohistochemical staining demonstrating the diffuse expression of neural cell adhesion molecule throughout the whole tumor (magnification, $\mathrm{x} 200$ ).

use) for detecting mouse $\operatorname{IgG}$, mouse $\operatorname{IgM}$ and rabbit primary antibodies) for $8 \mathrm{~min}$ at $37^{\circ} \mathrm{C}$. All samples were visualized using light microscopy.

To evaluate the multiple enlarged lymph nodes, Indium-111 octreotide scanning was performed, which indicated an increased uptake of multiple enlarged lymph nodes in the pre-sacral space at the 4 and 24-h delayed scans (Fig. 5). Multiple mesenteric lymph nodes in the right lower quadrant and left para-aortic lymph nodes exhibited no significant abnormal uptake, which suggested a reactive change accompanying acute enteritis.

A laparoscopic low anterior resection was performed, during which ovoid conglomerated enlarged mesorectal lymph nodes were identified and removed. The pathological data suggested that the NET had metastasized to 4 out of the 22 removed lymph nodes. There were no remnant tumors in 


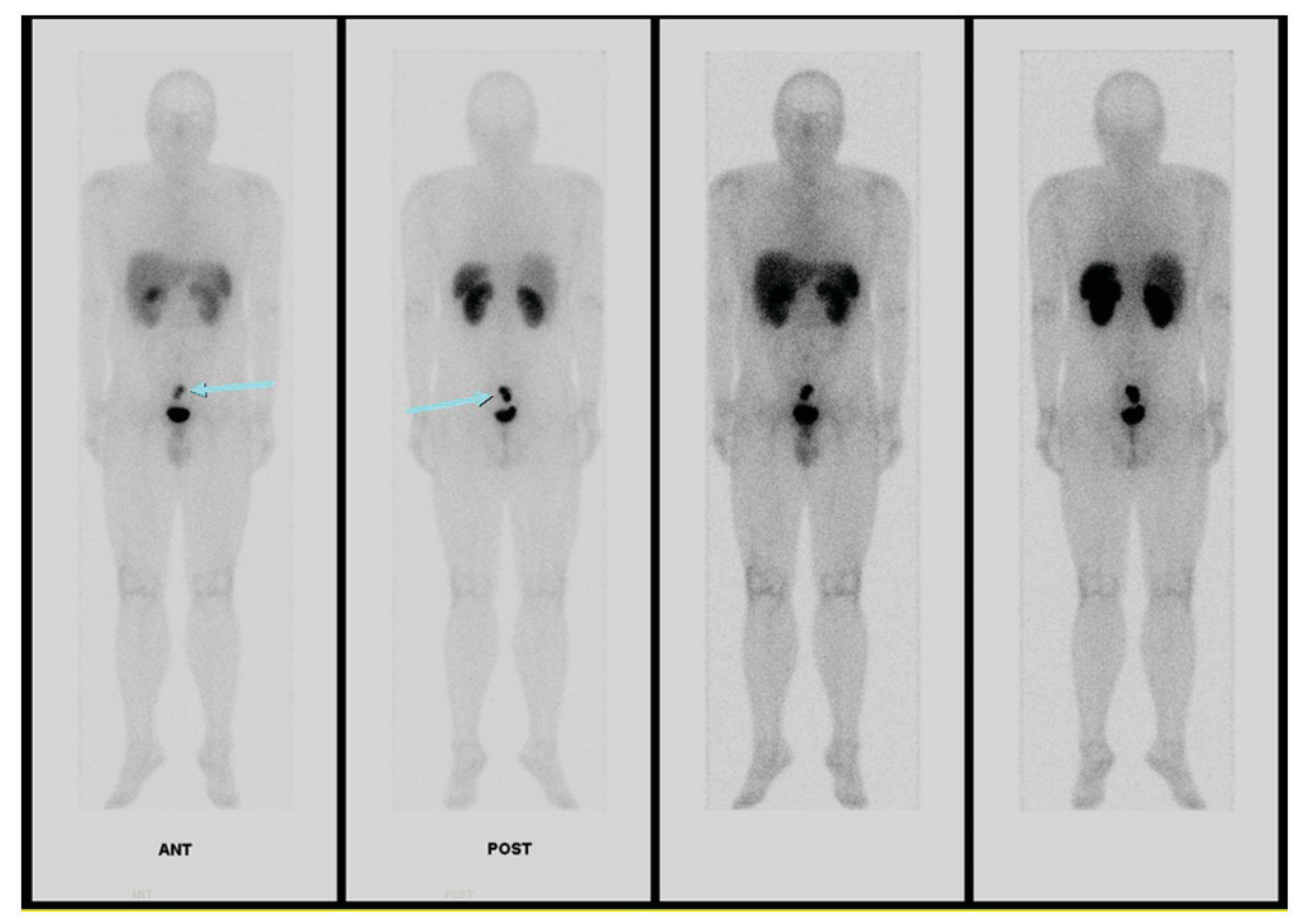

Figure 5. Indium-111 octreotide scanning indicating the increased uptake of multiple enlarged lymph nodes in the presacral space (arrow). There was no otherwise definite abnormal increased uptake in the abdomen. ANT, anterior; POST, posterior. The left two panels are images after a $4 \mathrm{~h}$ delay, and the right two panels are after a $24 \mathrm{~h}$ delay.

either the resected sigmoid colon or rectal tissues. The final diagnosis was rectal NET T1bN1M0 [stage IIIb, according to 2017 American joint Committee on Cancer Staging (12)]. A follow-up abdominopelvic computed tomography (CT) scan was performed 6 months after surgery was scheduled (December, 2017), which indicated no specific abnormal results. The patient was then scheduled for long-term ( $>5$ years) annual surveillance CT. Written informed consent was obtained from the patient.

\section{Discussion}

Rectal NETs are the most common type of gastroenteropancreatic NETs in Asia, accounting for 50\% of cases of gastroenteropancreatic NET (2). The majority of rectal NETs are asymptomatic, but may present with rectal bleeding or pain. If a rectal NET is suspected endoscopically (the typical finding is a yellowish-colored subepithelial tumor covered by normal mucosa), a rectal EUS is commonly performed to assess tumor size, depth of invasion and pararectal lymph nodes (13). The European Neuroendocrine Tumor Society Consensus guidelines recommend an imaging study (CT or magnetic resonance imaging) for tumors $>1 \mathrm{~cm}$ in size (13), whereas the North American Neuroendocrine Tumor Society Consensus guidelines do not routinely recommend cross-sectional radiographic studies for rectal NETs $<2 \mathrm{~cm}(4,14)$. If the tumor size is $>2 \mathrm{~cm}$, octreotide scintigraphy, useful for well-differentiated NETs, or positron emission tomography, useful for octreotide-negative tumors (including high-grade/poorly differentiated NETs), is recommended (13). Atypical endoscopic features, including semi-pedunculated morphology of the tumors, hyperemia, erosion or ulceration, are associated with tumor size and lymph node metastasis, requiring careful pretreatment evaluation $(15,16)$.

At present, the only established curative treatment for rectal NETs is complete resection. Rectal NETs of $<1 \mathrm{~cm}$ that are confined within the submucosa may be removed endoscopically due to the low risk of metastasis $(<3 \%)(13,17)$. However, other risk factors (mitotic rate $>2$ per $10 \mathrm{HPF}$ or lymphovascular invasion) should also be monitored following an endoscopic tumor resection, and if any known risk factors are identified, surgical resection should be considered. If lesions are $>2 \mathrm{~cm}$, the metastatic risk is high $(60-80 \%)$, and radical surgical resection with total mesorectal excision is required $(11,13)$.

In the case of tumors measuring between 1 and $2 \mathrm{~cm}$ in size, confined within the submucosa and lacking any of the aforementioned risk factors, there is disagreement about the optimal treatment modality (endoscopic or transanal resection vs. radical surgical resection); however, the endoscopic approach has been prioritized recently due to its minimal invasiveness and high rate of complete en bloc resection (18).

In the case of the present study, the young male patient had no known risk factors for metastasis, such as tumor size $(>2 \mathrm{~cm}$ ), endoscopic morphology involving ulceration, erosion or hyperemia, old age ( $>60$ years), WHO 2010 classification grade 3 disease (1), poorly differentiated histology, increased mitotic index, muscular propria invasion, lymphovascular invasion, angiogenesis or neural invasion $(13,19)$. However, significant lymph node metastasis was detected by CT imaging and octreotide scanning. Similarly, there have been specific case reports of small rectal carcinoid tumors with multiple liver or lymph node metastases (20-22), but those cases exhibited other known high-risk features despite the small tumor 
size, including central depression, high Ki-67 labeling index or lymphovascular invasion.

In summary, the present study described the case of a small rectal carcinoid tumor $(<2 \mathrm{~cm})$ with substantial lymph node metastasis without any known high-risk features. Therefore, the possibility of lymph node or distant metastasis must always be considered, even in cases of small rectal carcinoid tumors without any high-risk features. As highlighted in the present case, imaging studies, including CT or octreotide scanning, may be important additional tests for rectal NETs that appear benign without risk factors.

\section{Acknowledgements}

The authors would like to thank Dr Byong-Pil So from the Department of Pathology at Kangwon National University Hospital (Chuncheon, South Korea) for his consultation during the detailed process of pathological examination.

\section{Funding}

No funding was received.

\section{Availability of data and materials}

Data sharing is not applicable to this article, as no datasets were generated or analyzed during the present study.

\section{Authors' contributions}

SJ and SC performed the literature review, and wrote the manuscript. GB performed the surgery and discussed diagnosis and management with pathologists. SK performed pathological examination. CD and SJ performed diagnostic evaluation of the patient and corrected the manuscript.

\section{Ethics approval and consent to participate}

Written informed consent was obtained from the patient.

\section{Consent for publication}

Patient provided written informed consent for the publication of any associated data and accompanying images.

\section{Competing interests}

The authors declare that they have no competing interests.

\section{References}

1. Öberg K, Knigge U, Kwekkeboom D and Perren A; ESMO Guidelines Working Group: Neuroendocrine gastro-entero-pancreatic tumors: ESMO Clinical Practice Guidelines for diagnosis, treatment and follow-up. Ann Oncol 23 (Suppl 7): vii124-vii130, 2012.

2. Gastrointestinal Pathology Study Group of Korean Society of Pathologists; Cho MY, Kim JM, Sohn JH, Kim MJ, Kim KM Kim WH, Kim H, Kook MC, Park DY, et al: Current trends of the incidence and pathological diagnosis of gastroenteropancreatic neuroendocrine tumors (GEP-NETs) in Korea 2000-2009: Multicenter study. Cancer Res Treat 44: 157-165, 2012.
3. Ito T, Sasano H, Tanaka M, Osamura RY, Sasaki I, Kimura W, Takano K, Obara T, Ishibashi M, Nakao K, et al: Epidemiological study of gastroenteropancreatic neuroendocrine tumors in Japan. J Gastroenterol 45: 234-243, 2010.

4. Anthony LB, Strosberg JR, Klimstra DS, Maples WJ, O'Dorisio TM, Warner RR, Wiseman GA, Benson AB III and Pommier RF; North American Neuroendocrine Tumor Society (NANETS): The NANETS consensus guidelines for the diagnosis and management of gastrointestinal neuroendocrine tumors (nets): Well-differentiated nets of the distal colon and rectum. Pancreas 39: 767-774, 2010.

5. Jung YS, Yun KE, Chang Y, Ryu S, Park JH, Kim HJ, Cho YK, Sohn CI, Jeon WK, Kim BI and Park DI: Risk factors associated with rectal neuroendocrine tumors: A cross-sectional study. Cancer Epidemiol Biomarkers Prev 23: 1406-1413, 2014.

6. Hassan MM, Phan A, Li D, Dagohoy CG, Leary C and Yao JC: Risk factors associated with neuroendocrine tumors: A U.S.-based case-control study. Int J Cancer 123: 867-873, 2008.

7. Hemminki $\mathrm{K}$ and $\mathrm{Li} \mathrm{X}$ : Incidence trends and risk factors of carcinoid tumors: A nationwide epidemiologic study from Sweden. Cancer 92: 2204-2210, 2001

8. Takatsu Y, Fukunaga Y, Nagasaki T, Akiyoshi T, Konishi T, Fujimoto Y,NagayamaS and UenoM: Short-and Long-term outcomes of laparoscopic total mesenteric excision for neuroendocrine tumors of the rectum. Dis Colon Rectum 60: 284-289, 2017.

9. Kim JS, Kim YJ, Chung JW, Kim JH, Kim KO, Kwon KA, Park DK and An JS: Usefulness of endoscopic resection using the band ligation method for rectal neuroendocrine tumors. Intest Res 14: 164-171, 2016.

10. Konishi T, Watanabe T, Kishimoto J, Kotake K, Muto T and Nagawa H; Japanese Society for Cancer of the Colon and Rectum: Prognosis and risk factors of metastasis in colorectal carcinoids: Results of a nationwide registry over 15 years. Gut 56: 863-868, 2007.

11. Fahy BN, Tang LH, Klimstra D, Wong WD, Guillem JG, Paty PB, Temple LK, Shia J and Weiser MR: Carcinoid of the rectum risk stratification (CaRRs): A strategy for preoperative outcome assessment. Ann Surg Oncol 14: 1735-1743, 2007.

12. Chablaney S,Zator ZA and Kumta NA: Diagnosis and management of rectal neuroendocrine tumors. Clin Endosc 50: 530-536, 2017.

13. Caplin M, Sundin A, Nillson O, Baum RP, Klose KJ, Kelestimur F, Plöckinger U, Papotti M, Salazar R and Pascher A; Barcelona Consensus Conference participants: ENETS Consensus Guidelines for the management of patients with digestive neuroendocrine neoplasms: Colorectal neuroendocrine neoplasms. Neuroendocrinology 95: 88-97, 2012.

14. Kunz PL, Reidy-Lagunes D, Anthony LB, Bertino EM, Brendtro K, Chan JA, Chen H, Jensen RT, Kim MK, Klimstra DS, et al: Consensus guidelines for the management and treatment of neuroendocrine tumors. Pancreas 42: 557-577, 2013.

15. Shim KN, Yang SK, Myung SJ, Chang HS, Jung SA, Choe JW, Lee YJ, Byeon JS, Lee JH, Jung HY, et al: Atypical endoscopic features of rectal carcinoids. Endoscopy 36: 313-316, 2004.

16. Kim BN, Sohn DK, Hong CW, Han KS, Chang HJ, Jung KH, Lim SB, Choi HS, Jeong SY and Park JG: Atypical endoscopic features can be associated with metastasis in rectal carcinoid tumors. Surg Endosc 22: 1992-1996, 2008.

17. Onozato Y, Kakizaki S, Iizuka H, Sohara N, Mori M and Itoh H: Endoscopic treatment of rectal carcinoid tumors. Dis Colon Rectum 53: 169-176, 2010.

18. Chen T, Yao LQ, Xu MD, Zhang YQ, Chen WF, Shi Q, Cai SL, Chen YY, Xie YH, Ji Y, et al: Efficacy and safety of endoscopic submucosal dissection for colorectal carcinoids. Clin Gastroenterol Hepatol 14: 575-581, 2016.

19. de Mestier L, Brixi H, Gincul R, Ponchon T and Cadiot G: Updating the management of patients with rectal neuroendocrine tumors. Endoscopy 45: 1039-1046, 2013.

20. Yamagishi D, Matsubara N, Noda M, Yamano T, Tsukamoto K, Kuno T, Hamanaka M, Kobayashi M, Ikeuchi H, Matsuda I, et al: Clinicopathological characteristics of rectal carcinoid patients undergoing surgical resection. Oncol Lett 4: 910-914, 2012.

21. Akasu T, Moriya Y and Sugihara K: Transrectal ultrasonography of a small rectal carcinoid tumor with lymph node metastasis: A case report. Jpn J Clin Oncol 26: 112-115, 1996.

22. Shin EK, Park SJ, Kim KJ, Moon W, Moo In Park, Dong Han Im, Lee JS and Park CB: A case of a small rectal carcinoid tumor with multiple liver metastasis. Clin Endosc 38: 103-106, 2009.

This work is licensed under a Creative Commons Attribution-NonCommercial-NoDerivatives 4.0 International (CC BY-NC-ND 4.0) License. 